\title{
Microstructural asymmetry of the corticospinal tracts predicts right-left differences in circle drawing skill in right-handed adolescents
}

\author{
Steffen Angstmann ${ }^{1}$ (D) Kathrine Skak Madsen ${ }^{1} \cdot$ Arnold Skimminge $^{1} \cdot$ \\ Terry L. Jernigan ${ }^{1,2} \cdot$ William F. C. Baaré ${ }^{1}$ Hartwig Roman Siebner ${ }^{1,3}$
}

Received: 11 September 2015/Accepted: 19 December 2015/Published online: 11 January 2016

(c) The Author(s) 2016. This article is published with open access at Springerlink.com

\begin{abstract}
Most humans show a strong preference to use their right hand, but strong preference for the right hand does not necessarily imply a strong right-left asymmetry in manual proficiency (i.e., dexterity). Here we tested the hypothesis that intra-individual asymmetry of manual proficiency would be reflected in microstructural differences between the right and left corticospinal tract (CST) in a cohort of 52 right-handed typically-developing adolescents (11-16 years). Participants were asked to fluently draw superimposed circles with their right dominant and left non-dominant hand. Temporal regularity of circle drawing movements was assessed for each hand using a digitizing tablet. Although all participants were right-handed, there was substantial inter-individual variation regarding the relative right-hand advantage for fluent circle drawing. All subjects underwent whole-brain diffusion tensor imaging at 3 Tesla. The right and left CST were defined as regions-of-interest and mean fractional anisotropy (FA) and diffusivity values were calculated for right and left CST. On average, mean FA values were higher in the left CST relative to right CST. The degree of right-left FA asymmetry showed a linear relationship with right-left asymmetry in fluent circle drawing after correction for age
\end{abstract}

Steffen Angstmann

steffena@drcmr.dk

1 Danish Research Centre for Magnetic Resonance, Centre for Functional and Diagnostic Imaging and Research, Copenhagen University Hospital Hvidovre, 2650 Hvidovre, Denmark

2 Center for Human Development, University of California, San Diego, CA, USA

3 Department of Neurology, Copenhagen University Hospital Bispebjerg, Bispebjerg, Denmark and gender. The higher the mean FA values were in the left dominant CST relative to the right non-dominant CST, the stronger was the relative right-hand advantage for regular circle drawing. These findings show that right-left differences in manual proficiency are highly variable in righthanded adolescents and that this variation is associated with a right-left microstructural asymmetry of the CST.

Keywords Manual dexterity - Brain development - Circle drawing · Corticospinal tract · Hemispheric asymmetry · Diffusion tensor imaging

\section{Introduction}

Humans have extraordinary ability to perform skilled and well-coordinated hand movements, which enables them to solve demanding manual tasks. This often involves the manipulation of an object, such as in writing or drawing. The high level of dexterity in humans and non-human primates has been linked to the existence of fast-conducting monosynaptic connections in the pyramidal tract that link the motor cortex to the spinal motor neurons supplying the hand muscles (Lemon 1999). These monosynaptic corticospinal projections enable independent finger movements, an ability that is fundamental to dexterity (Lemon 2008).

Another intriguing feature of manual motor control is that most humans show a clear preference towards using one hand for skilled movements. This striking example of a behavioral asymmetry is called handedness or dextrality. A series of brain imaging studies have been conducted to identify functional or structural asymmetries in the motor system that can be attributed to preferential hand use. Functional neuroimaging studies have consistently shown 
functional inter-hemispheric asymmetries in motor cortical regions during manual motor tasks depending on the individual degree of handedness (Dassonville et al. 1997; Volkmann et al. 1998; Kloppel et al. 2007).

At the macrostructural level, $T_{1}$-weighted structural magnetic resonance imaging (MRI) has revealed an anatomical fingerprint of handedness in the human central sulcus. The central sulcus was found to be deeper in the hemisphere contralateral to the dominant hand in both right-handers and left-handers (Amunts et al. 2000). Using shape analysis, it was shown that the human hand knob, a major landmark of the hand motor representation, is located more dorsally in the left hemisphere in right-handers than in left-handers (Sun et al. 2012). These handednessrelated differences in sulcus depth or shape are not captured by more traditional voxel-based methods investigating differences between left-handers and right-handers. Indeed, voxel-based morphometry (VBM) applied to $T_{1^{-}}$ weighted MRI of large cohorts of healthy adults failed to reveal consistent inter-hemispheric structural asymmetries related to handedness (Good et al. 2001; Watkins et al. 2001). Another VBM study only found a statistical trend towards differences in right-left asymmetry, when comparing the asymmetry maps of young right-handed $(n=56)$ and left-handed $(n=56)$ men (Herve et al. 2006).

Other studies have used diffusion-weighted imaging (DWI) to investigate handedness related structural asymmetry in the corticospinal tract (Gong et al. 2005; Westerhausen et al. 2007; Li et al. 2010; Seizeur et al. 2013). DWI is sensitive to the diffusion of water molecules and provides information about microstructural properties of tissue, because diffusion of water molecules depends on the structural properties of encompassing tissue. In white matter, diffusion is less hindered parallel than perpendicular to the fiber bundles, because of the axonal membranes as well as the surrounding myelin sheaths and other microstructural characteristics. This explains why voxels in the white matter show tract-dependent anisotropic behavior. Diffusion properties can be characterized by applying a diffusion tensor model at the voxel level (Basser et al. 1994). Fractional anisotropy (FA) and mean diffusivity (MD) are well-established diffusion tensor-based metrics of regional water diffusion in a given voxel. The FA value indicates how much water diffusion has a directional bias, whereas the MD value describes the direction-independent overall magnitude of diffusion in the voxel (Basser et al. 1994). In addition, diffusivity can also be expressed as either parallel $\left(\lambda_{\|}\right)$or perpendicular $(\lambda \perp)$ to the principal diffusion direction (Beaulieu 2009, 2002).

In children, diffusion tensor imaging (DTI) has shown age-related increases in FA and decreases in MD in multiple white matter tracts including the CST (Eluvathingal et al. 2007; Lebel et al. 2008). These age-related microstructural DTI changes provide evidence for continuing maturation of white matter tracts into early adulthood (Lebel et al. 2008). In healthy adults, DTI consistently showed inter-hemispheric asymmetries with higher FA and lower MD values in the CST of the left hemisphere. With the exception of an early DTI study (Buchel et al. 2004), handedness had no influence on the individual expression of the left $>$ right FA asymmetry in the adult brain (Gong et al. 2005; Westerhausen et al. 2007; Li et al. 2010; Seizeur et al. 2013). Handedness is a multidimensional construct that comprises many facets and aspects (Healey et al. 1986; Janssen 2004) and hand preference tests, such as the Edinburgh Handedness Inventory (Oldfield 1971), do not consider right-left asymmetries in manual proficiency (i.e., dexterity). Therefore, previous DTI studies might have yielded negative results because they tested for an association between microstructure and handedness rather than manual dexterity. Interestingly, a recent study in a small sample of 14 adults, employing DWI tractography, observed that manual dexterity in grip strength and fingertapping performance was associated with a left higher than right FA asymmetry of respectively the CST and intrahemispheric pre- and post central gyrus connections (Rose et al. 2012).

In the present study we used DTI to test the hypothesis that the individual expression of dexterity is represented in the microstructure of the main corticospinal pathway, which conveys cortical commands to the hand. We tested this hypothesis in typically-developing right-handed adolescents because CST maturation as indexed by DTI measures is still ongoing in this period of life. We only included dextral individuals with strong preference for the right hand because we were interested to identify microstructural asymmetry in right and left CST related to motor proficiency beyond preferred hand use. To this end, participants were asked to perform a circle-drawing task with the right and left hand, respectively, while drawing movements were recorded with a digitizing tablet (Henkel et al. 2001; Siebner et al. 2002). A kinematic measure of unimanual proficiency would better reflect right-left asymmetry in manual ability than handedness questionnaires, which capture differences in preferred hand use, but not skill, across a range of motor tasks (Rose et al. 2012).

\section{Materials and methods}

\section{Subjects}

Sixty-five typically-developing right-handed children and adolescents from three schools in the Copenhagen suburban area participated in the study. All participants were 
enrolled in a longitudinal study to prospectively trace developmental changes, called the "HUBU" project ("HUBU" = "Hjernens Udvikling hos Børn og Unge"Brain maturation in children and adolescents). Participants were examined every 6 months, both behaviorally and in the MR scanner. Data used in the present study were collected in the eighth assessment round of the HUBU project. A detailed description of the HUBU cohort is given elsewhere (Madsen et al. 2010, 2011; Vestergaard et al. 2011; Klarborg et al. 2013). Exclusion criteria were any known history of neurological or psychiatric disorders or significant brain injury according to parent reports. According to the Edinburgh Handedness Inventory (Oldfield 1971), participants were consistently right-handed with handedness scores ranging from 44 to 100 (median score: 100). Prior to participation and after receiving oral and written explanation of the study aims and study procedures, all children assented to the procedures and informed written consent was obtained from the parents or guardians of all subjects. The study was approved by the local Danish Committee for Biomedical Research Ethics (H-KF-01-131/ 03 ) and conducted in accordance with the Declaration of Helsinki.

Of the 65 right-handed individuals who participated in the eighth assessment, 13 subjects were excluded because of the following reasons: no MRI acquired due to dental braces $(n=8)$, poor MR-image quality $(n=2)$, technical problems with the behavioral task $(n=1)$, incidental findings on MRI $(n=1)$ and parent reported psychiatric disorder $(n=1)$. Thus, the final sample consisted of 52 right-handed individuals (18 males and 34 females). The mean age was 13.7 years (standard deviation $=1.75$, range: $11-16$ years).

\section{Circle drawing task}

Subjects performed a simple circle drawing task with their right and left hands (Fig. 1a). Participants performed the circle drawing task with the ulnar part of the hand resting on the table. This ensured that circle drawing only involved coordinated movements of the hand and finger, while the forearm maintained a constant position. Participants were asked to continuously draw concentric superimposed circles at a convenient speed. They were informed that we were mainly interested in testing how well they can produce circles in a regular fashion. Therefore, we emphasized that they should try to smoothly produce consecutive circles at a constant speed. To facilitate fluent open-loop performance, participants were told that it was not necessary to ensure that one circle exactly overlay the other. First, they drew circles with their right dominant hand in clockwise direction for $10 \mathrm{~s}$. Thereafter, participants drew circles with their left hand in counter-clockwise direction for $10 \mathrm{~s}$, producing the homologous movement pattern as in the right-hand task. Because of the simplicity of the task, no test runs were performed.

A pressure sensitive digitizing tablet (WACOM Intuos4 large PTK-840, Wacom Technology Corporation, Vancouver, WA, USA) recorded the writing trace that was produced by the tip of the pen at a sample rate of $200 \mathrm{~Hz}$. Kinematics of the writing movements were analyzed with commercial software (CS-Win, version 10.05, MedCom, Munich, Germany).

\section{Kinematic analysis of circle drawing}

The vertical drawing traces and the corresponding velocity profiles produced with the right and left hand were automatically segmented into consecutive up-and-down strokes. Curves of vertical velocity were calculated and smoothed by nonparametric regression methods (Fig. 1b), (Marquardt and Mai 1994). We were mainly interested in assessing the temporal regularity of drawing movements. Therefore, we chose the variability of peak vertical velocity during circle drawing as the kinematic measure of interest and calculated the coefficient of variance $(\mathrm{CV}=$ standard deviation/mean $)$ for peak drawing velocity. The higher the $\mathrm{CV}$, the less proficient were the circle drawing movements.

The CV of peak vertical drawing velocity was calculated separately for right hand drawing $\left(\mathrm{CV}_{\mathrm{R}}\right)$ and left hand drawing $\left(\mathrm{CV}_{\mathrm{L}}\right)$. We then calculated a laterality index $\left(\mathrm{LI}_{\mathrm{CV}}\right)$ as an asymmetry measure for manual proficiency, using the following formula.

$\mathrm{LI}_{\mathrm{CV}}=\frac{\mathrm{CV}_{\mathrm{R}}-\mathrm{CV}_{\mathrm{L}}}{\mathrm{CV}_{\mathrm{R}}+\mathrm{CV}_{\mathrm{L}}} \times(-100)$

Values between -100 and -1 revealed a higher proficiency of circle drawing for the left hand which produced more regular velocity profiles than the right hand. Conversely, values between 1 and 100 indicate a right-hand advantage for regular circle drawing. Figure 1 shows the parameterization of the $\mathrm{LI}_{\mathrm{CV}}$.

\section{Magnetic resonance imaging}

All subjects were scanned using a 3T Siemens Magnetom Trio MR scanner (Siemens, Erlangen, Germany) with an eight-channel head coil (Invivo, Fl, USA). All acquired scans were aligned parallel to the anterior-posterior commissural line. High-resolution 3D $T_{1}$-weighted images of the whole head were acquired using a MPRAGE sequence $(\mathrm{TR}=1550 \mathrm{~ms}, \mathrm{TE}=3.04 \mathrm{~ms}$, matrix $256 \times 256,192$ sagittal slices with no gap, $1 \mathrm{~mm}^{3}$ isotropic voxels). Whole brain diffusion-weighted (DW) images were acquired using a twice-refocused balanced spin echo sequence that 
Fig. 1 Recording of circle drawing and calculation of the laterality indices $\left(\mathrm{LI}_{\mathrm{CV}}\right)$. a Subjects were required to draw concentric superimposed circles on a digitizing tablet with their right dominant or left non-dominant hand. b Left and right hand traces were segmented into sequential upand down-strokes and $\mathbf{c}$ the coefficient of variance (CV) of velocity stroke peaks $(\mathrm{CV})$ was calculated separately for circle drawing movements with the right or left hand. d A laterality index $\left(\mathrm{LI}_{\mathrm{CV}}\right)$ was calculated based on the mean $\mathrm{CV}$ of the right and left hand $\mathrm{CV}$. e Histogram depicting the distribution of the $\mathrm{LI}_{\mathrm{CV}}$ in the 52 participants (age range: 11-16 years)
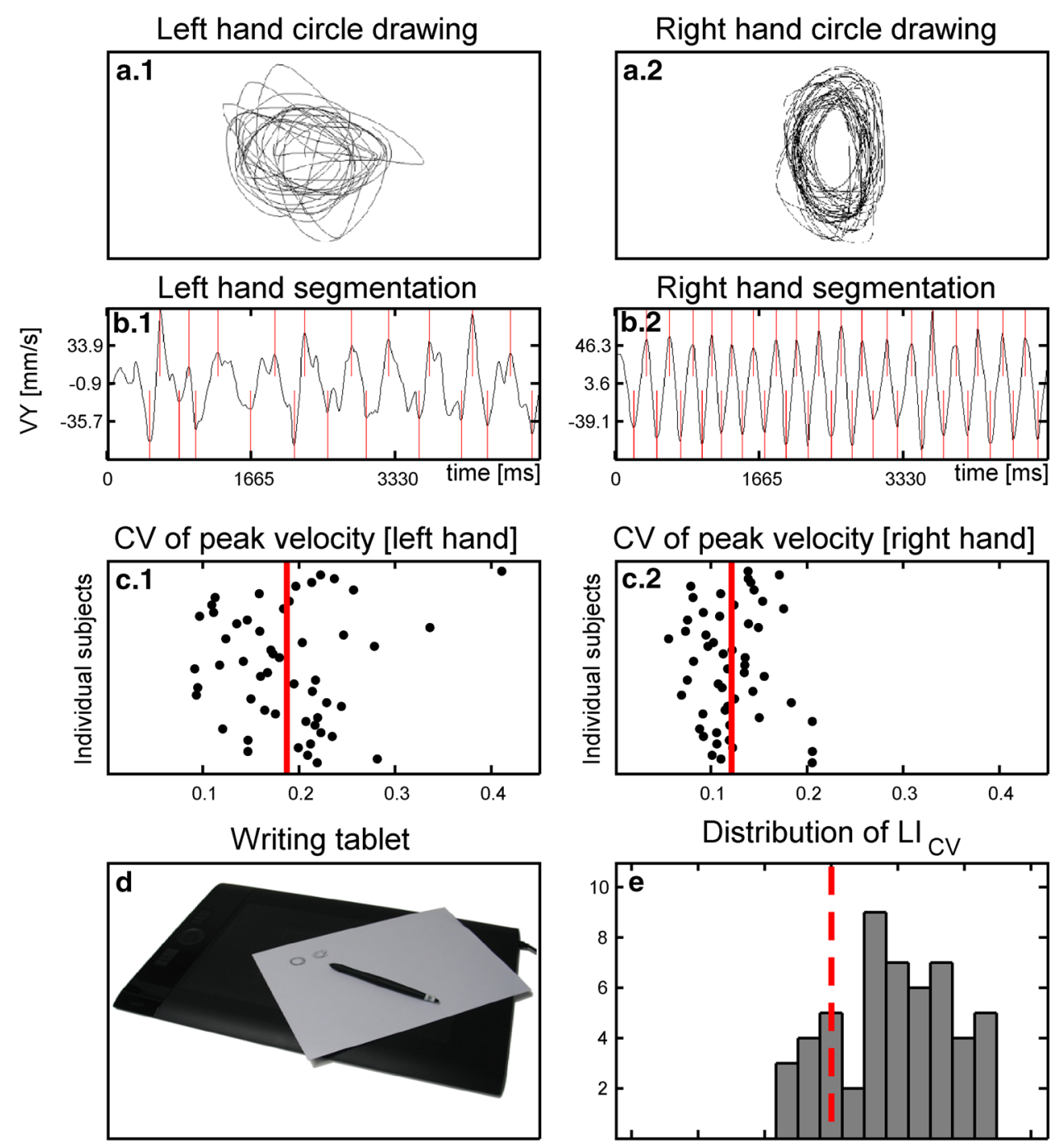

minimized eddy current distortion (Reese et al. 2003). Ten non-DW images $(b=0)$ and 61 DW images $(b=1200 \mathrm{~s} /$ $\mathrm{mm}^{2}$ ), encoded along independent collinear diffusion gradient orientations, were acquired $(\mathrm{TR}=8200 \mathrm{~ms}$, $\mathrm{TE}=100 \mathrm{~ms}, \quad \mathrm{FOV}=220 \times 220, \quad$ matrix $=96 \times 96$, GRAPPA: factor $=2,48$ lines, 61 transverse slices with no gap, $2.3 \mathrm{~mm}^{3}$ voxels). A gradient echo field map was acquired to correct b0 field distortions $(\mathrm{TR}=530 \mathrm{~ms}$, $\mathrm{TE}[1]=5.19 \mathrm{~ms}$ and $\mathrm{TE}[2]=7.65 \mathrm{~ms}, \mathrm{FOV}=256 \times$ 256; matrix $=128 \times 128,47$ transverse slices with no gap, voxel size $=2 \times 2 \times 3 \mathrm{~mm}^{3}$ )

\section{Image pre-processing and analysis}

Prior to analysis and blind to behavioral data, raw images from all subjects were visually inspected to ascertain sufficient image quality. Images were preprocessed using pipelines implemented in MATLAB, using mainly SPM 8 (Wellcome Department of Cognitive Neurology, University College London, UK) routines. $T_{1}$-weighted images were corrected for spatial distortions due to non-linearity in the gradient system of the scanner (Jovicich et al. 2006) and oriented to the MNI coordinate system using a sixparameter mutual information rigid transformation. Subsequently, the mean b0 image was co-registered to the $T_{1}$ weighted image using a six-parameter mutual information rigid transformation after which all diffusion-weighted images were co-registered (no reslicing) to the mean b0 image. Next, all co-registered images were corrected for geometric distortions using a voxel displacement map based on both the acquired b0 field map (Andersson et al. 2001) and the scanner-specific gradient non-linearity 
(Jovicich et al. 2006). Finally, all images were resliced into the MNI coordinate system using tri-linear interpolation. Note that this procedure involves only one re-slicing step. The diffusion gradient orientations were adjusted to account for any rotation applied during registration. The diffusion tensor was fitted using a least-squares-fit by nonlinear optimization employing a Levenburg-Marquardt algorithm and constrained to be positive definite by fitting its Cholesky decomposition as embedded in Camino. Fractional anisotropy (FA), mean diffusivity [MD $=\left(\lambda_{1}+\right.$ $\left.\lambda_{2}+\lambda_{3}\right) / 3$ ] as well as diffusivity parallel $\left(\lambda_{\|}=\lambda_{1}\right)$ and perpendicular $\left[\lambda \perp=\left(\lambda_{2}+\lambda_{3}\right) / 2\right]$ to the principal diffusion direction were calculated. A brain mask based on the realigned and distortion corrected b0 images was applied to the FA and diffusivity images.

\section{Inter-subject spatial normalization of fiber tracts}

All DTI measures of interest (i.e., FA, MD, $\lambda_{\|}$and $\lambda \perp$ ) were extracted from regions-of-interest (ROIs) to test specific hypotheses and to determine the anatomical specificity of observed associations (see below). Spatial normalization and alignment of fiber tracts across subjects was achieved using the Tract-Based Spatial Statistics (TBSS) module, part of FSL 4.1 .4 (Smith et al. 2006), which was used to project DTI measures onto a mean FA skeleton that represents the centers of all tracts common to the group. As we were interested in investigating right-left difference in CST microstructure, we generated a symmetric skeleton.

At first, subjects' FA images were aligned into a common space using the non-linear registration tool FNIRT (Andersson et al. 2007). A study-specific target, the groups' most representative FA image, was then identified after non-linearly registering each subjects FA image to every other subjects FA image. Next, the target FA image was aligned to MNI space using affine registration and subsequently the entire aligned dataset was transformed into $1 \mathrm{~mm}^{3}$ MNI space. A cross-subject mean FA image was created and thinned to create a mean FA skeleton, representing the centers of all tracts common to the group. The mean FA skeleton was thresholded at FA $>0.25$ and contained $134.184 \mathrm{~mm}^{3}$ interpolated voxels, corresponding to approximately one quarter of the voxels with FA above 0.25 . The aligned FA images of each subject were then projected onto the mean skeleton by locating the highest local FA value in the direction perpendicular to the skeleton tracts and assigning this value to the skeleton. Next we generated the symmetric skeleton. First, a symmetric mean FA map was generated by averaging the mean FA map and a (left-right) flipped version of the mean FA image. The symmetric mean FA map was thinned, and masked with a (one voxel) dilated version of the original mean FA skeleton, ensuring that only skeleton segments that were almost symmetric in the original non-symmetric skeleton were included. Finally, the preliminary symmetric skeleton was masked with its left-right flipped counterpart to generate a symmetric mean skeleton, which contained 112.616 voxels. In addition, the non-linear warps, skeleton projections and symmetric skeleton generation were applied to the MD, $\lambda_{\|}$and $\lambda_{\perp}$ data.

\section{Regions-of-interest (ROIs)}

ROIs were drawn onto the symmetric skeleton overlaid on a symmetric mean FA image in FSLview. The primary motor and sensory hand area was taken as the cortical origin for the CST containing the corticospinal connections supplying the hand muscles. This was in accordance with findings by (Kumar et al. 2009) who demonstrated in a sample of adolescents that the CST originated both from pre- and postcentral gyri. The hand knob in the motor cortex was identified (Yousry et al. 1997) and the white matter underlying the hand knob and the white matter underlying the corresponding region in the sensorimotor cortex was included in our CST ROIs. Below the level of the corpus callosum, information from the probabilistic fiber atlas implemented in FSLview (Hua et al. 2008) was used to delineate the corticospinal tract ROIs down through the posterior limb of the internal capsule. Manual finetuning of the ROIs was guided by the superior-inferior direction (blue) in the target's color-coded FA map and a white matter atlas (Mori et al. 2005). No voxels were included inferior to MNI slice $z=-14$. Figure 2 shows the ROIs of the CST. Each ROI contained 1.785 voxels.

FA data from both the left and the right hemisphere were extracted separately. As for the kinematic data, we calculated a laterality index for the FA data $\left(\mathrm{LI}_{\mathrm{FA}}\right)$ using the formula.

$\mathrm{LI}_{\mathrm{FA}}=\frac{\sum_{\mathrm{CST}_{\mathrm{R}}} \mathrm{FA}-\sum_{\mathrm{CST}_{\mathrm{L}}} \mathrm{FA}}{\sum_{\mathrm{CST}_{\mathrm{R}}} \mathrm{FA}+\sum_{\mathrm{CST}_{\mathrm{L}}} \mathrm{FA}} \times 100$

Values between -100 and -1 reflect higher FA values in the left CST, while values between 1 and 100 indicate higher FA values in the right CST. LIs were also calculated for MD, $\lambda_{\|}$and $\lambda_{\perp}$. To be able to control for global hemispheric left-right asymmetries we calculated the same asymmetry index for all skeleton voxels in the left vs. the right hemisphere.

\section{Statistical analysis and visualization}

Statistical analyses were performed using SPSS software (SPSS 19, IBM, Somers, NY, USA). Independent and dependent variables did not significantly deviate from the Gaussian distribution as ascertained with the Shapiro-Wilk test. Effects of age and gender on circle-drawing 
Fig. 2 The upper panels show the ROIs of the corticospinal tract (marked in red) and the symmetric TBSS skeleton (marked in yellow). The ROIs and the skeleton are overlaid on corresponding axial, coronal and sagittal slices of a group map displaying mean FA values in stereotactic space. The lower panel depicts a smoothed 3D visualization of the ROIs and their position within the standard MNI-152 template

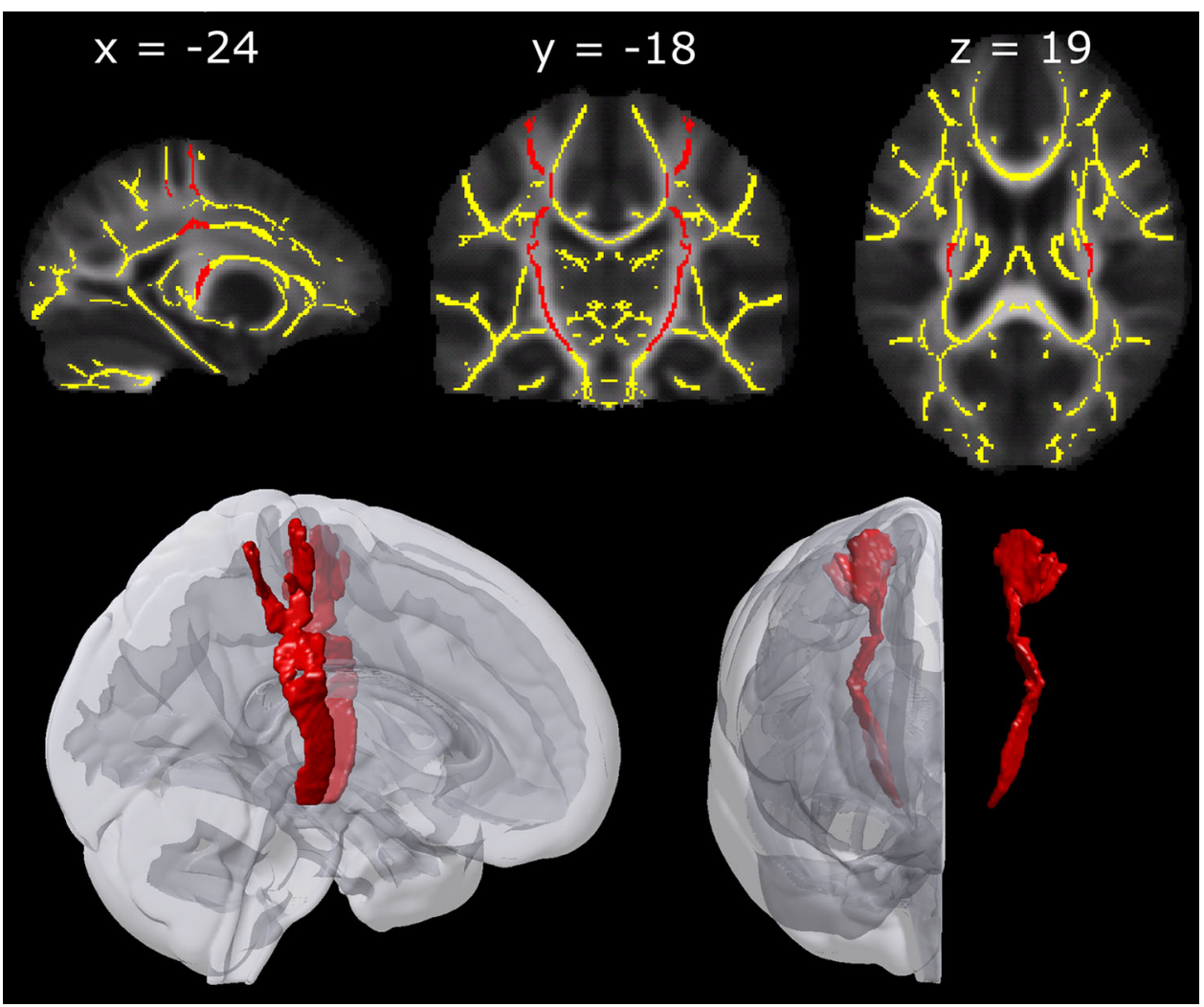

performance, FA and MD values were examined using linear regressions. Laterality in the behavioral and ROI measures was examined using multiple linear regression models controlling for age and gender.

All assumptions for linear regressions were met and multiple linear regression tests were performed hierarchically. Our main hypotheses were that individual differences in the degree of laterality of drawing stability (i.e. laterality index $\mathrm{LI}_{\mathrm{CV}}$ ) would be associated with the degree of laterality in CST FA and MD, after adjusting for age and gender. These hypotheses were tested employing a twosided statistical threshold of 0.025 (Bonferroni corrected for two tests as we tested for two diffusion parameters).

Planned follow-up analyses were contingent on observing significant associations with FA and MD in our primary analyses. First, to assess anatomical specificity of observed associations, we additionally factored whole hemisphere white matter asymmetry as a nuisance regressor into the analyses. Second, to assess the relative contribution of the left and right CST, we modeled left and right CST FA both as simultaneous and as separate predictors of $\mathrm{LI}_{\mathrm{CV}}$, after adjusting for age and gender. To further explore the nature of observed FA effects, we applied the same analysis to $\lambda_{\|}$and $\lambda \perp$, as these measures may give additional information about the underlying microstructural indices. Follow-up analyses were considered significant when $p \leq 0.05$.
To obtain further anatomical information about the lateralization of the CST and its relation to behavioral asymmetry on a smaller spatial scale, we performed two additional steps of analyses. We calculated the laterality indices for each axial slice $\left(\mathrm{LI}_{\mathrm{FA} \text {-slice }}\right)$ in each subject without correction for gender and age to identify topographical variations in the expression of right-left differences along the CST. Slicewise calculations were performed for each of the 85 axial slices along the $z$-axis of MNI stereotactic space, from slice $z=-14$ to $z=70$. Based on these $85 \mathrm{LI}_{\mathrm{FA} \text {-slice }}$-indices of our 52 participants, a one-sample permutation test was performed (100.000 permutations) in MATLAB to test which parts of the CST showed significant asymmetry in FA values. The tests were done using an approach originally developed for EEG-data (Blair and Karniski 1993) and implemented in MATLAB (Groppe et al. 2011). This approach relies on point-wise permutations of series of data and a $t_{\max }$ statistic. We employed an overall family-wise error rate of $\alpha=0.05$. Moreover, we calculated uncorrected slice-wise correlations between $\mathrm{LI}_{\mathrm{FA} \text {-slice }}$ and $\mathrm{LI}_{\mathrm{CV}}$ in order to provide complementary information about the regional distribution of the association between the degree of FA-laterality and the degree of behavioral laterality throughout the course of the CST ROI. For this exploratory follow-up analysis, no correction for gender and age was performed.

In addition, we performed a complementary voxel-based analysis on the whole white matter skeleton of the 
association between the right-left voxel-wise difference and $\mathrm{LI}_{\mathrm{CV}}$, controlling for age and gender to test whether our findings were specific for the CST or whether other brain regions also showed similar effects. To do so, we employed the randomize module in FSL (Nichols and Holmes 2002) using 10.000 permutations. Significant associations were determined using the threshold-free cluster enhancement method (TFCE) (Smith and Nichols 2009) optimized for 2D-like white matter skeletons with a corrected threshold of $p<0.05$. Finally, an effect-size map is presented to provide further anatomical information about the effects outside the CST. The effect-size map is a $t$-map of the association between the right-left voxel-wise difference and $\mathrm{LI}_{\mathrm{CV}}$, controlling for age and gender.

\section{Results}

\section{Circle drawing}

Most participants showed a right-hand advantage for regular circle drawing, producing less variable peak velocities with their right dominant hand than with the left nondominant hand (Fig. 3c). Although all participants were consistent right-handers, the relative right-hand advantage for fluent circle drawing varied considerably across participants. Some subjects showed no clear difference in $\mathrm{CV}$ of peak velocity between the right and left hand or even a slight left-hand advantage, whereas others showed a clear right-hand advantage (Fig. 1e).

Analyses revealed that variability of circle drawing velocity linearly decreased with age (Fig. 3a, b). This agedependent improvement in temporal stability of circle drawing was comparable for the right dominant and left non-dominant hand. Accordingly, the laterality index $\mathrm{LI}_{\mathrm{CV}}$ reflecting the right-left asymmetry of circle drawing performance did not change as a function of age (Fig. 3c). We found no gender effects on $\mathrm{LI}_{\mathrm{CV}}(\beta=0.074, p=0.602)$. There was a statistical trend towards a significant interaction between age and gender for left-hand circle drawing $(\beta=-0.26, p=0.062)$ and the circle drawing laterality index $(\beta=-0.264, p=0.059)$, caused by a trend towards an earlier improvement in temporal regularity of left-hand circle drawing with age in boys relative to girls.
Fig. 3 Scatter plots showing the linear relationship between circle drawing $(\mathbf{a}-\mathbf{c})$, FA values $(\mathbf{d}-\mathbf{f}$,$) and \mathrm{MD}$ values $(\mathbf{g}-\mathbf{i})$ and age of the participants. Regressions are calculated separately for left $(\mathbf{a}, \mathbf{d}, \mathbf{g})$, right $(\mathbf{b}, \mathbf{e}, \mathbf{h})$ and laterality indices $(\mathbf{c}$, f, i). Data are linearly interpolated with a black line and regression coefficient $(\beta)$ and significance level $(p)$ are given in the upper right corner of the respective plot
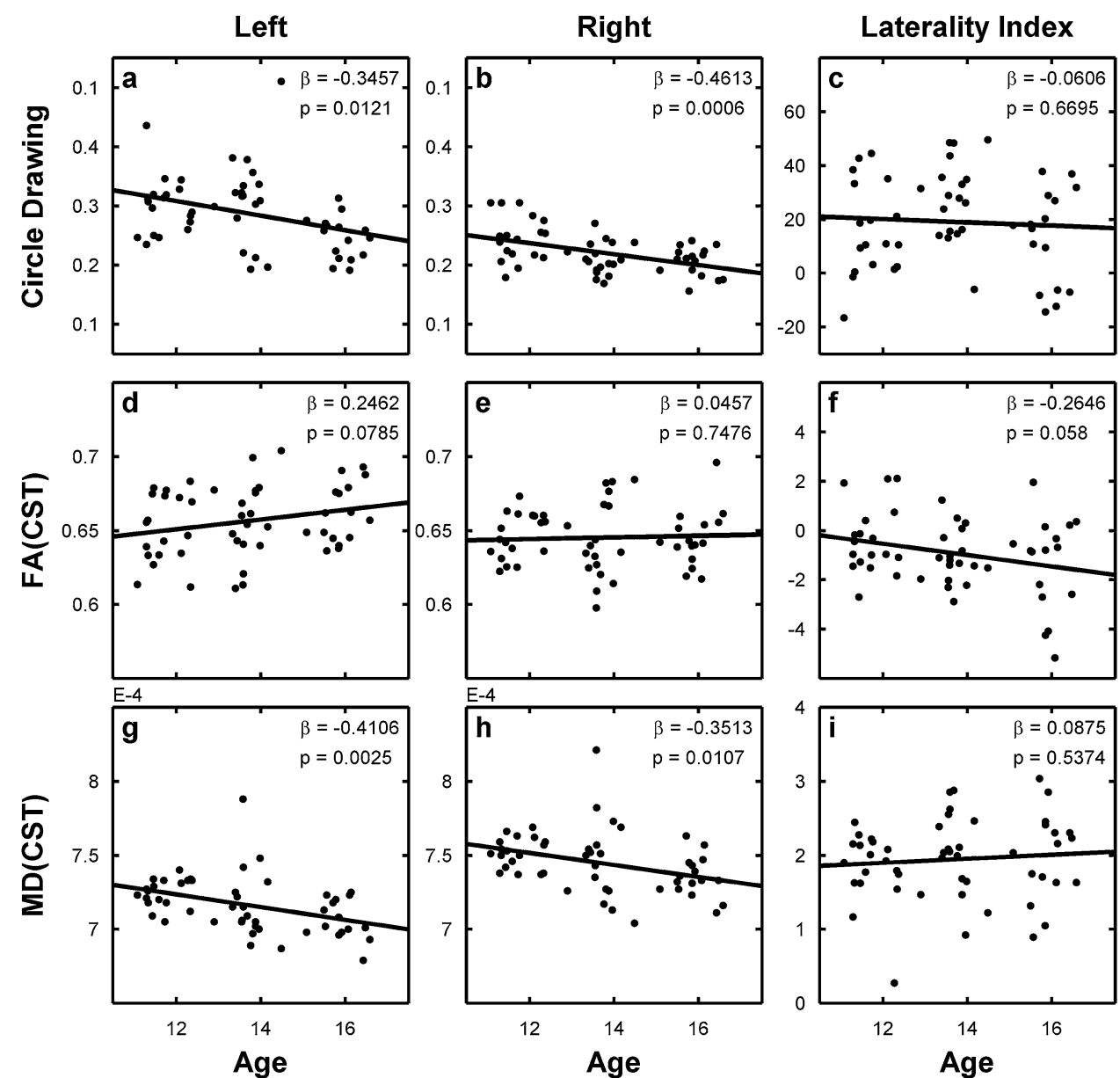


\section{Microstructural asymmetry of the corticospinal tract (CST)}

In the majority of our right-handed participants, mean FA values in the left CST were higher than in the right CST (Fig. 3f), resulting in a negative right-left laterality index for FA $\left(\mathrm{LI}_{\mathrm{FA}}\right)$. Yet, intra-individual asymmetry of mean FA in the CST varied substantially across participants, and more than $20 \%$ of the participants had a positive $\mathrm{LI}_{\mathrm{FA}}$. There was a trend towards a linear increase of FA values with age in the left CST $(\beta=0.246, p=0.079$; Fig. $3 \mathrm{~d})$, but not in the right CST $(\beta=0.046, p=0.748$; Fig. $3 \mathrm{e})$. Accordingly, the $\mathrm{LI}_{\mathrm{FA}}$ also tended to become more negative with age $(\beta=-0.265, p=0.058$; Fig. $3 f)$. Mean FA in right CST was higher in males than females $(\beta=-0.28$, $p=0.044)$. This was not the case for left CST $(b=-0.17$, $p=0.22)$.

Mean MD in the left and right CST showed a significant linear decrease with age (Fig. 3g, h). Lower MD values were observed in the left relative to the right CST, but the left-right relation remained unchanged across the age range $(\beta=0.09, p=0.537$, Fig. $3 i)$. None of the DTIbased asymmetry indices showed any significant effect for gender (all $p$ values $>0.25$ ) or any age by gender interaction (all $p$ values $>0.13$ ).

\section{Relation between asymmetry in dexterity and CST microstructure}

The multiple regression model confirmed our primary hypothesis that the asymmetry in mean FA between the right and left CST ( $\mathrm{LI}_{\mathrm{FA}}$ ) was a significant positive predictor of right-left asymmetry in drawing performance as indexed by $\mathrm{LI}_{\mathrm{CV}}$ (Table 1, model 1 and Fig. 4a). The stronger the right $<$ left FA asymmetry in CST (i.e., the lower $\mathrm{LI}_{\mathrm{FA}}$ ), the stronger was the relative right-hand advantage for fluent circle drawing (i.e., the higher was the $\mathrm{LI}_{\mathrm{CV}}$ ). Adjusting for hemispheric asymmetry in white matter FA values between right and left hemisphere did not change our results (Table 1, model 1b). Right-left asymmetry of mean MD in the CST did not predict right-left differences in drawing performance (Table 1, model 2).

The results of the main analysis motivated planned follow-up analyses to further explore the nature of the effects (see Table 2). We investigated FA values in left and right CST both separately and simultaneously and applied

Table 1 Linear regression models predicting circle drawing laterality $\left(\mathrm{LI}_{\mathrm{cv}}\right)$ with right-left asymmetry of fractional anisotropy (FA) and mean diffusivity (MD) in the ROI covering the corticospinal tract

\begin{tabular}{|c|c|c|c|c|c|c|c|c|c|c|}
\hline \multirow[t]{2}{*}{ Model } & \multirow[t]{2}{*}{$R^{2}$} & \multirow[t]{2}{*}{ DTI measure } & \multicolumn{2}{|l|}{ LI } & \multicolumn{2}{|l|}{ Age } & \multicolumn{2}{|c|}{ Gender } & \multicolumn{2}{|l|}{$\mathrm{WH}_{\mathrm{LI}}$} \\
\hline & & & $\beta$ & $p$ & $\beta$ & $p$ & $\beta$ & $p$ & $\beta$ & $p$ \\
\hline $1^{\mathrm{a}}$ & 0.118 & FA & -0.343 & 0.019 & -0.154 & 0.278 & 0.047 & 0.730 & - & - \\
\hline $1^{\mathrm{b}}$ & 0.147 & FA & -0.371 & 0.012 & -0.127 & 0.372 & 0.041 & 0.762 & 0.175 & 0.218 \\
\hline $2^{\mathrm{a}}$ & 0.011 & MD & 0.020 & 0.892 & -0.063 & 0.671 & 0.074 & 0.618 & - & - \\
\hline
\end{tabular}

Significant results with $p<0.05$ are highlighted in bold

$W H_{L I}$ Whole hemisphere laterality index

a Models not controlled for whole hemisphere FA asymmetry

b Models controlled for whole hemisphere FA asymmetry

Fig. 4 Partial regression plots predicting behavioral asymmetry $\left(\mathrm{LI}_{\mathrm{CV}}\right)$ as a function of corticospinal tract asymmetry $\left(\mathrm{LI}_{\mathrm{FA}}\right)$ in figure (a) and left and right $\mathrm{FA}$ values simultaneously but as separate regressors (b). Regressions are adjusted for age and gender
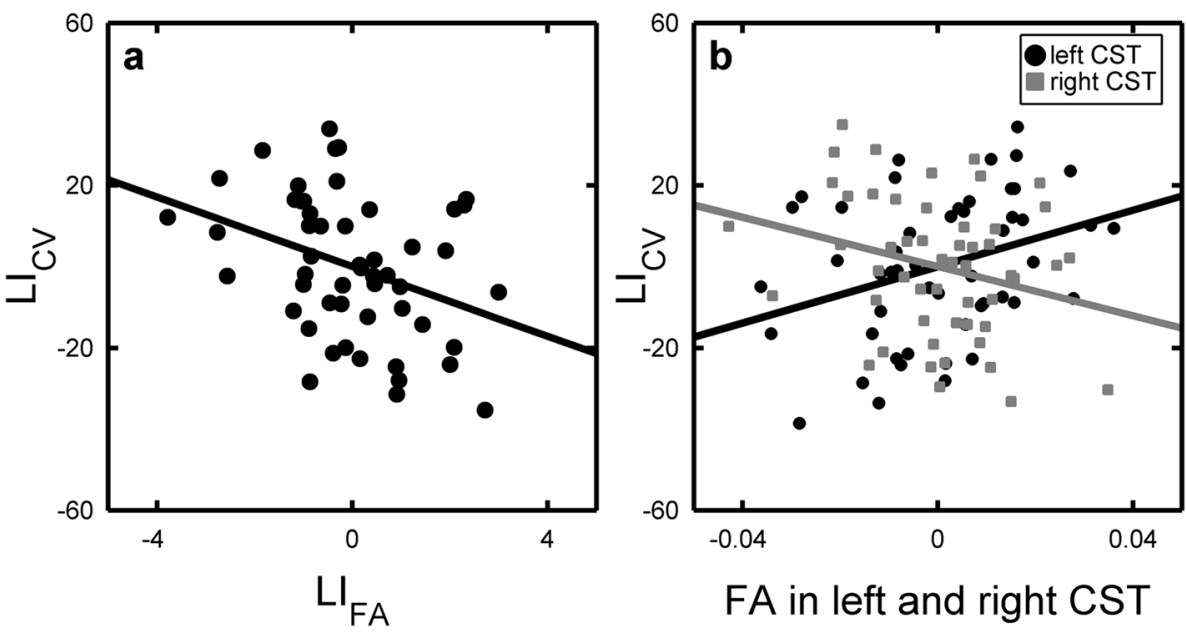
Table 2 Linear regression models (follow-up models) predicting circle drawing laterality $\left(\mathrm{LI}_{\mathrm{cv}}\right)$ with diffusivities in the left and right corticospinal tract, both simultaneously and separately

\begin{tabular}{|c|c|c|c|c|c|c|c|c|c|c|}
\hline \multirow[t]{2}{*}{ Model } & \multirow[t]{2}{*}{$R^{2}$} & \multirow[t]{2}{*}{ DTI measure } & \multicolumn{2}{|c|}{ Left ROI } & \multicolumn{2}{|c|}{ Right ROI } & \multicolumn{2}{|l|}{ Age } & \multicolumn{2}{|c|}{ Gender } \\
\hline & & & $\beta$ & $p$ & $\beta$ & $p$ & $\beta$ & $p$ & $\beta$ & $p$ \\
\hline $3^{\mathrm{a}}$ & 0.121 & FA & 0.462 & 0.020 & -0.361 & 0.064 & -0.162 & 0.265 & 0.063 & 0.661 \\
\hline $4^{\mathrm{b}}$ & 0.233 & FA & 0.222 & 0.139 & - & - & -0.123 & 0.404 & 0.120 & 0.407 \\
\hline $5^{\mathrm{b}}$ & 0.113 & FA & - & - & -0.058 & 0.702 & -0.062 & 0.670 & 0.062 & 0.683 \\
\hline $6^{\mathrm{a}}$ & 0.372 & $\lambda_{\|}$ & 0.484 & 0.013 & -0.428 & $\mathbf{0 . 0 3 7}$ & -0.134 & 0.372 & 0.103 & 0.453 \\
\hline $7^{\mathrm{b}}$ & 0.230 & $\lambda_{\|}$ & 0.215 & 0.145 & - & - & -0.021 & 0.884 & 0.104 & 0.466 \\
\hline $8^{\mathrm{b}}$ & 0.126 & $\lambda_{\|}$ & - & - & -0.086 & 0.587 & -0.099 & 0.529 & 0.071 & 0.623 \\
\hline $9^{a}$ & 0.319 & $\lambda_{\perp}$ & -0.542 & 0.034 & 0.459 & 0.066 & -0.147 & 0.326 & 0.067 & 0.643 \\
\hline $10^{\mathrm{b}}$ & 0.184 & $\lambda_{\perp}$ & -0.169 & 0.279 & - & - & -0.124 & 0.418 & 0.114 & 0.437 \\
\hline $11^{\mathrm{b}}$ & 0.104 & $\lambda_{\perp}$ & - & - & 0.037 & 0.813 & -0.057 & 0.702 & 0.068 & 0.653 \\
\hline
\end{tabular}

Significant results with $p<0.05$ are highlighted in bold

a Models including the left and right ROI simultaneously as predictors of $\mathrm{LI}_{\mathrm{CV}}$, controlling for age and gender

${ }^{b}$ Models including the left and right ROI as separate predictors of $\mathrm{LI}_{\mathrm{CV}}$, controlling for age and gender the same analysis to $\lambda_{\|}$and $\lambda \perp$, as these measures might give additional information about the underlying microstructural indices. When modeled as simultaneous predictors of $\mathrm{LI}_{\mathrm{CV}}$, mean FA in both the right and left CST contributed significantly to the prediction of right-left differences in drawing performance (Table 2, model 3 and Fig. 4b): Mean FA values in left CST showed a positive linear relationship with $\mathrm{LI}_{\mathrm{CV}}$. Conversely, mean FA values in right CST showed a negative relation with $\mathrm{LI}_{\mathrm{CV}}$. In addition, diffusivity parallel $\left(\lambda_{\|}\right)$and diffusivity perpendicular $\left(\lambda_{\perp}\right)$ to the main axis of the diffusion tensor model exhibited significant associations with $\mathrm{LI}_{\mathrm{CV}}$. Left CST $\lambda_{\|}$ was positively associated with right-left asymmetry in drawing performance, whereas right CST $\lambda_{\|}$showed a negative linear relationship with $\mathrm{LI}_{\mathrm{CV}}$. An opposite pattern emerged for the left CST, where left CST $\lambda_{\perp}$ displayed a negative linear relationship with $\mathrm{LI}_{\mathrm{CV}}$, whereas right CST $\lambda_{\perp}$ showed a trend towards a positive linear relation with $\mathrm{LI}_{\mathrm{CV}}$. Importantly, this was only true when the DTI-based measures were modeled simultaneously, but not separately (Table 2, $b$ models). Together, the results show that it was not the microstructure of either the left or right CST alone that accounted for the left-right asymmetry in circle drawing. The results rather show that it is the relation between the microstructural properties of the right and left CSTs, which is associated with asymmetry between left and right hand circle drawing abilities.

\section{Slice-wise expression of structural right-left asymmetry}

Slice-wise spatial expression of right $<$ left asymmetry in FA varied considerably along the CST (Fig. 5). Permutation analysis revealed several clusters of slices where the right $<$ left asymmetry in mean FA reached significance at the group level, whereas such asymmetry did not reach significance in other portions of the CST (Fig. 5b). The slice-wise expression of the linear relationship between right-left asymmetry in FA ( $\left.\mathrm{LI}_{\mathrm{FA} \text {-slice }}\right)$ and drawing skill $\left(\mathrm{LI}_{\mathrm{CV}}\right.$ ) also varied substantially along the CST (Fig. 5c). The CST sections showing the strongest association between $\mathrm{LI}_{\mathrm{FA} \text {-slice }}$ and $\mathrm{LI}_{\mathrm{CV}}$ were clustered around the axial slices at $z=10$ (corresponding to the internal capsule), $z=27$ (corresponding to the superior corona radiata), and slice $z=49$, which is a complex region containing fibers from different tracts, e.g. corpus callosum, CST and superior longitudinal fasciculus. Of note, the slices showing the strongest right $<$ left asymmetry in FA values were not necessarily those slices, where the degree of right $<$ left asymmetry predicted a right-hand advantage for circle drawing. Figure $5 \mathrm{~d}$ depicts the position of the ROI in the MNI $z$-slices -14 to 70 .

\section{Voxel-wise analysis of the entire white-matter skeleton}

We also performed a complementary voxel-wise analysis on the whole white matter skeleton. It revealed two significant clusters, displaying a linear relationship between left-right FA asymmetries and asymmetry in circle drawing (Fig. 6). Both clusters were located in the CST; one at the level of the superior corona radiata and the other one in about the same complex region as found in the slice-wise analysis. Both of them are positioned in our ROI and no voxels outside the CST reached significance after correction for multiple comparisons.

Based on the whole skeleton analysis, we generated an effect-size map showing the regional distribution of the association between $\mathrm{LI}_{\mathrm{CV}}$ and left-right $\mathrm{FA}$ asymmetry in the brain (Fig. 7). The symmetric TBSS-skeleton 


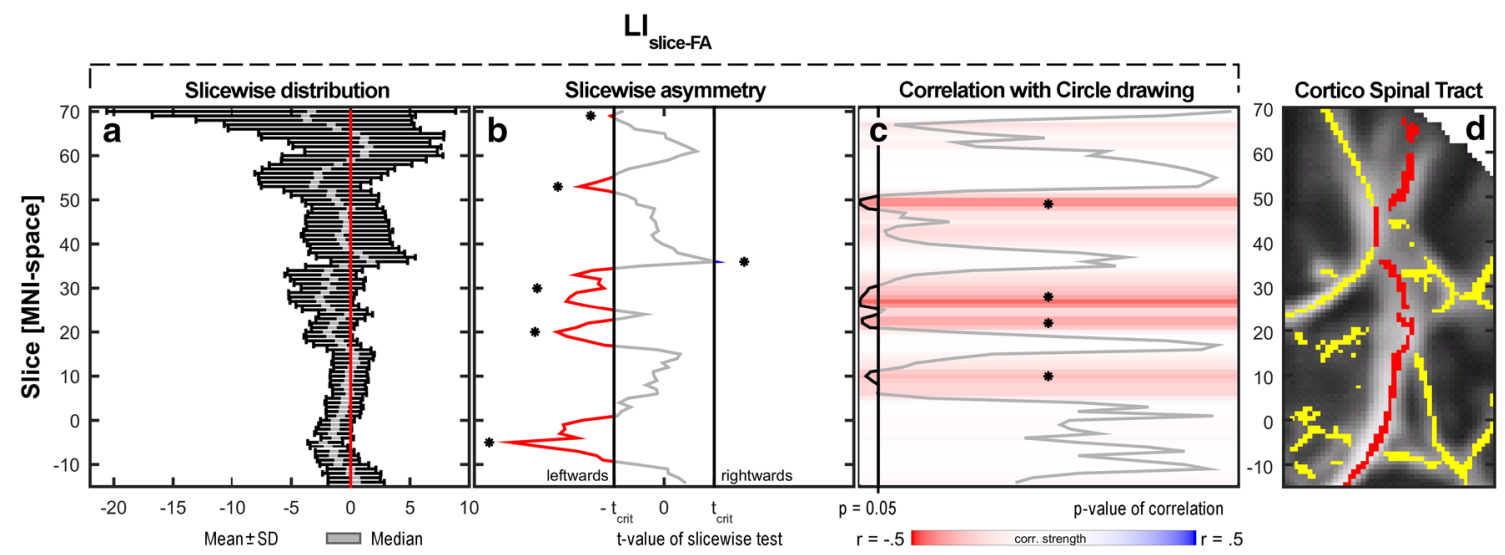

Fig. 5 Slice-wise expression of the right-left asymmetry in mean FA $\left(\mathrm{LI}_{\mathrm{FA}-\mathrm{slice}}\right)$ in axial slices of the corticospinal tract from $z=-14$ to $z=70$ in MNI-space. a Distribution of $\mathrm{LI}_{\mathrm{FA} \text {-slice }}$ values in the whole sample. For each slice are shown means and standard deviations as black bars overlaid by the median in grey. b Slice-wise asymmetry as assessed with a permutation test (corrected for multiple comparisons). The red-grey-blue line indicates $t$-values. The vertical black lines indicate corrected, two-tailed threshold criteria of significance; $t$ values not contained between these are significantly different from

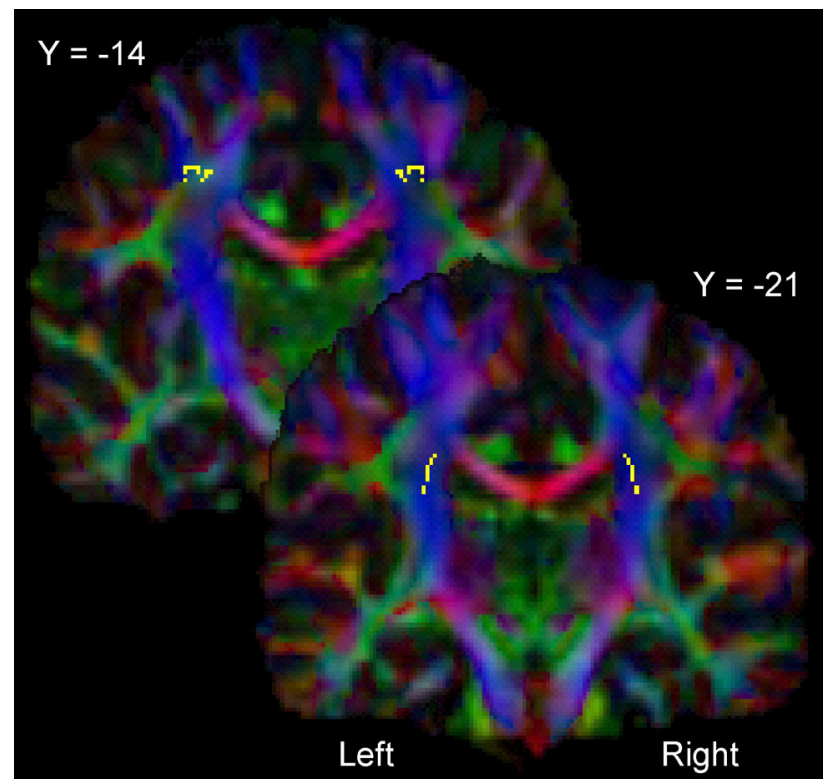

Fig. 6 Statistical parametric maps showing those voxels where the right-left difference in FA shows a significant linear relationship with the right-left asymmetry in circle drawing as indexed by $\mathrm{LI}_{\mathrm{CV}}$ ( $p<0.05$, correction for multiple comparisons). The voxels are presented as axial slice overlaid on the color-coded group FA map. The color-coding depicts the direction of the primary eigenvectors of the diffusion tensor in each voxel as follows: anterior-posterior in green, superior-inferior in blue and left-right in red. The two slices shown are MNI slice $y=-21$ (corona radiata) and MNI slice $y=-14$, depicting a complex region containing crossing fibers

comprised a total of 56.308 voxels per hemisphere of which 1.713 (3.04\%) had a $t$-value above 2.0106 $(p \leq 0.05$, uncorrected), while $1.278(2.27 \%)$ had a zero, i.e. asymmetric, and marked in red (leftwards) or blue (rightwards). c Slice-wise correlations (uncorrected for age and gender) with the writing laterality index $\mathrm{LI}_{\mathrm{CV}}$. Color of the slice indicates correlation strength ( $r$-value); black line indicates $p$ value of the correlation test. d Partial coronal slice (at $y=-18$ ) showing the skeleton (yellow) overlaid on the mean FA map from $z=-14$ to $z=70$. The red color indicates the part of the skeleton included in the CST-ROI

$t$-value below -2.0106 ( $p \leq 0.05$ uncorrected). This corresponds to $5.31 \%$ of the total number of voxels in the skeleton which would be expected to occur by chance. Within the pre-defined CST ROI (1.785 voxels), this summed percentage was $9.47 \%$ (169 voxels). In accordance with the main result based on left-right FA asymmetry of the entire CST, the vast majority of the significant voxels showed a positive linear relation between $\mathrm{LI}_{\mathrm{CV}}$ and left-right FA asymmetry. 163 out of 1785 voxels $(9.13 \%)$ had a $t$-score $>2.0106$, whereas only six voxels $(0.34 \%)$ had a $t$-score $<-2.0106$.

\section{Discussion}

This is to the best of our knowledge the first study linking a functional right-left asymmetry in manual skill to a microstructural right-left asymmetry of the corticospinal tract (CST) in the adolescent brain. In typically-developing adolescents, we combined fine-grained kinematic measurements to characterize the regularity of circle drawing movements and non-invasive brain DTI imaging to measure FA and MD in the CST. We found that higher FA values in the left relative to the right CST were associated with higher proficiency in right-hand circle drawing relative to left-hand circle drawing. The individual expression of left $>$ right FA differences in CST predicted how much the right hand outperformed the left hand in terms of producing temporally stable velocity profiles in a simple circle drawing task. This link between right-left asymmetry in CST microstructure and circle drawing skill was not 


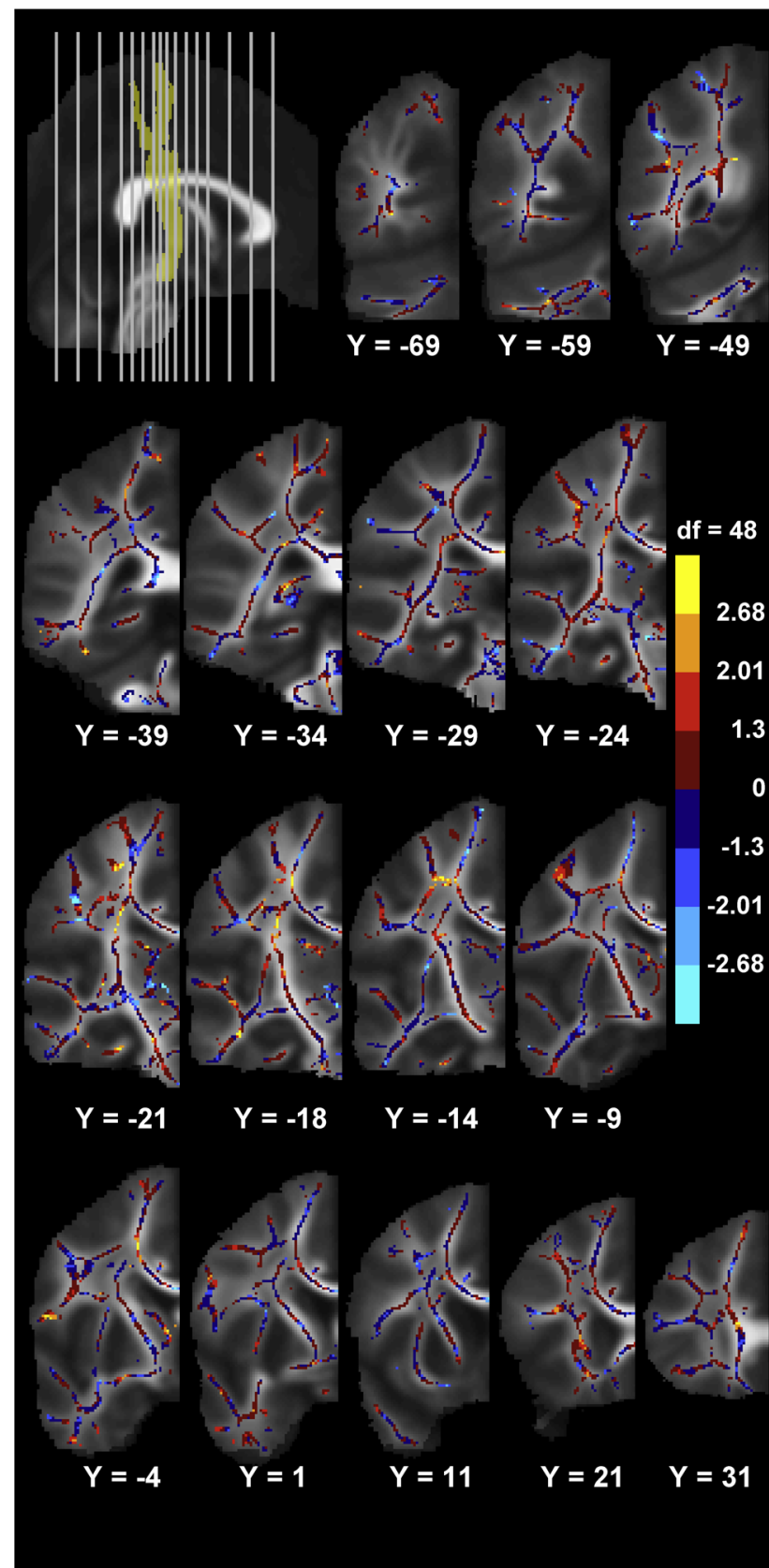

Fig. 7 Effect-size map of the association between the circle drawing laterality index $\mathrm{LI}_{\mathrm{CV}}$ and the right-left voxel-wise difference in FAvalues, corrected for age and gender. The effect-size map is overlaid on the mean symmetric FA images. Only one hemisphere is visualized as a difference score is investigated. The association of higher right-left voxel-wise FA difference with better right-to-left circle drawing performance is shown in warm colors. Negative values are shown in cool colors. The color bar shows the color mapping of all skeleton voxels to the values of the uncorrected $t$-statistics $(t=0$, $p=1 ; t= \pm 1.229, p=0.2 ; t= \pm 2.01, p=0.05 ; t= \pm 2.682$, $p=0.01)$. The MNI coordinates for the coronal images are given under each image. In the upper left corner the position of the coronal slices shown are depicted on the mid-sagittal image with the position of the CST schematized. Coronal slice -69 is displayed to the far left and 31 to the far right on the mid-sagittal image driven by inter-individual differences in preferred hand use, because all participants were consistent right-handers.

The microstructural properties of the right and left CST jointly contributed to the right-left difference in fluent circle drawing. When modeled simultaneously, FA in the right and left CST contributed significantly to the prediction of individual right-left differences in drawing performance, but not when modeled separately. In the simultaneous model, mean FA values in left CST showed a positive linear relationship with $\mathrm{LI}_{\mathrm{CV}}$, whereas mean FA values in right CST showed a negative relation with $\mathrm{LI}_{\mathrm{CV}}$. Our results are in line with those of a recent study in a small sample of 14 adults showing significant associations between manual dexterity in grip strength and finger-tapping performance and left higher than right FA asymmetry of respectively the CST and intrahemispheric connections between the pre- and post central gyrus (Rose et al. 2012). Together, these results strongly indicate that asymmetry indices based on a direct comparison of FA in corresponding white matter pathways of the left and right hemisphere, as compared to regional FA values in a single hemisphere, are better suited to identify structural correlates of lateralized function in the human brain. This notion is also supported by animal work showing the relevance of microstructural asymmetries between homologous areas for lateralized brain function (Ocklenburg and Güntürkün 2012).

The present study was designed to identify a microstructural correlate of right-left differences in manual ability (i.e., dexterity). We only included dextral adolescents with a clear preference for using the right hand during common daily manual tasks. While the focus on consistently dextral individuals minimized the confounding effects of preferential hand use, the present study provides no information about the relation between preferred hand use (i.e. handedness or dextrality) and microstructural asymmetry of the corticospinal tract. Indeed it would be very interesting to assess this function-structure relationship in left-handed individuals. Another open question is how much the degree of handedness modulates the relationship between the right-left asymmetry in manual skilfulness and the microstructural asymmetry in the corticospinal tract. These questions need to be addressed in future studies.

Using a different MRI-based microstructural marker, Hervé et al. (2009) studied the effects of handedness and dexterity on structural asymmetry of CS in a large sample of adolescents $(n=409)$, aged from 12 to 18 years. The CST appears relatively hypointense on $T_{1}$-weighted MRIs which has been attributed to the presence of large corticospinal axons (Barkovich, 2000; Yagishita et al., 1994). Taking advantage of the relation between $T_{1}$-weigted sig- 
nal intensity of the CST and density of large-calibre CST axons, Hervé et al. (2009) performed a ROI analysis to compare "apparent grey matter density" (aGMd) in the putative CST in the posterior part of the internal capsule. They found a strong left $>$ right hemispheric aGMd asymmetry in adolescents which was less pronounced in individuals with preferred left hand use (Hervé et al. 2009). In apparent contrast to our present results, left $>$ right hemispheric aGMd asymmetry did not reflect inter-individual variations in manual proficiency as indexed by the Grooved Pegboard task (Hervé et al. 2009). Since the DTIderived FA measure might reflect slightly different properties of the CST than the $T_{1}$-derived aGMd measure, FA might be more sensitive than aGMd to reveal a relation between right-left asymmetry in CST microstructure and dexterity. The discrepancy might also be related to differences in the type of task used to determine asymmetry in dexterity. Our circle drawing task probed the temporal stability of muscular synergies of distal finger muscles, while the pegboard task used by Hervé et al. (2009) did probe a combined grasping-aiming skill involving distal (grasping) and proximal (aiming) synergies.

Taken together, the work by Herve et al. (2009) and the present study suggest that the microstructure of the CST in the adolescent brain is related to both preferred hand use and skill level, but different MRI-based microstructural markers might be more closely related to handedness or dexterity. The question how well various MRI measures of CST microstructure capture functional right-left asymmetries in relation to handedness or dexterity remains to be systematically studied in a multi-modal MRI study. Since the relationship between CST microstructure and motor hand function might change during brain maturation, a longitudinal study covering the transition from childhood to adulthood is warranted.

When comparing the microstructure of right and left CST, asymmetry in mean FA but not mean diffusivity predicted the right-left difference in fluent circle drawing. In other words, the directionality of regional water diffusion in the CST rather than its direction-independent magnitude of water diffusion reflected the right-left asymmetry in dexterity. FA values are considered to reflect axonal density, diameter, myelination and organization, and fiber coherence within a given voxel and are taken as an index of structural connectivity. A number of DTI studies have linked regional FA variation in white matter tracts with inter-individual performance differences in tasks probing specific cognitive abilities or skills (Wolbers et al. 2006; Madsen et al. 2010; Vestergaard et al. 2011; Madsen et al. 2011). Regional FA values in tracts linking different brain regions have been shown to be related to functional connectivity in a way that increased structural connectivity predicts increased functional connectivity
(Damoiseaux and Greicius 2009; Groppa et al. 2012; Jung et al. 2012).

The regional FA value is determined by perpendicular $\left(\lambda_{\perp}\right)$ and parallel $\left(\lambda_{\|}\right)$diffusivity. Therefore, the relationship between right-left asymmetry in FA and circle drawing might have been caused by an asymmetry in $\lambda_{\perp}$, $\lambda_{\|}$, or both. We found that $\lambda_{\|}$in left CST was positively associated with right-left asymmetry in drawing performance (as indexed by $\mathrm{LI}_{\mathrm{CV}}$ ), whereas $\lambda_{\|}$in right CST showed a negative linear relationship with $\mathrm{LI}_{\mathrm{CV}}$. Conversely, $\lambda_{\perp}$ in left CST displayed a negative linear relationship with $\mathrm{LI}_{\mathrm{CV}}$, whereas $\lambda_{\perp}$ of the right CST showed a trend towards a positive linear relation with $\mathrm{LI}_{\mathrm{CV}}$. We therefore conclude that DTI-based measures of perpendicular $\left(\lambda_{\perp}\right)$ and parallel $\left(\lambda_{\|}\right)$diffusivity jointly contributed to the asymmetry in mean FA between the left and right CST and hereby, to right-left differences in regular circle drawing. This raises the question how regional diffusion properties of water, as derived from DTI, relate to the histological features of the CST, such as axonal density, diameter and myelination, which determine its neurophysiological function (Hartline and Colman 2007). In a mouse model, increased regional $\lambda_{\perp}$ values coincided with the extent of demyelination and were distinct from axonal damage (Song et al. 2005). In infants, it has been found that FA and $\lambda_{\perp}$ but not $\lambda_{\|}$are structural markers of myelination and that changes in these two measures are closely related to functional improvement in electrical conduction efficiency (Dubois et al. 2008). Specifically, increased electrical conduction speed is positively correlated with FA values and negatively correlated with $\lambda_{\perp}$ values. As mentioned previously, the large and heavily myelinated corticospinal axons make monosynaptic connections with cervical motor neurons supplying the hand and are thought to be critical to manual dexterity (Lemon 1999, 2008). Therefore, it is tempting to speculate that a higher number of very large corticospinal axons along with increased myelination might cause higher FA values in the CST contralateral to the more proficient hand. Yet it is important to bear in mind that the neuroanatomical interpretation of DTI-based metrics is not straightforward. A number of other tissue properties such as axonal density, axonal diameter, extracellular volume fraction as well as crossing fibers and tract geometry determine water diffusion in addition to the degree of myelination and the range of axonal diameters in the CST (Gulani et al. 2001; Beaulieu 2002; Mukherjee et al. 2002; Hasan and Narayana 2006; Jito et al. 2008).

The main analysis was a ROI analysis based on the mean FA value of all voxels in the TBSS skeleton belonging to the CST. This ROI approach lacked topographic specificity within the CST. Yet we were interested to know whether the microstructural right-left asymmetry 
in FA was expressed homogeneously throughout the CST or whether the expression of microstructural asymmetry varied along the CST. To this end, we computed slice-wise effect size maps which consisted of axial slices along the CST (Fig. 5c). The slice-wise map pinpointed three sections of the CST where the right-left asymmetry in FA was particularly pronounced, including a voxel cluster in the internal capsule, superior corona radiata and a complex region containing fibers from different tracts, e.g. corpus callosum, CST and superior longitudinal fasciculus. The latter two clusters were linked to manual dexterity not only on slice level, but also on single-voxel level after correction for multiple comparisons. The spatially variable expression of right-left asymmetry in FA along the CST can at least partially be explained by the fact that many voxels that are assigned to the CST in the TBSS skeleton contain fibers from other tracts that cross the CST and hereby may attenuate the effect size of microstructural CST asymmetry. These tracts serve different functions and feature their own characteristics. In particular, tracts related to language functions exhibit asymmetry themselves (Vernooij et al. 2007; Friederici 2009), which is not related to motor proficiency. For example, the corona radiata comprises the corticospinal tract, the corticopontine tract and the corticobulbar tract. Callosal fibers and fibers of long sagittal fiber tracts blend with corticospinal axons in the respective voxels. Furthermore, the corticopontine tract and the superior thalamic radiation are situated close to each other and both tracts might influence the regional FA values. Within the CST, the corticospinal projections are not only targeting the hand. This implies that those voxels containing the corticospinal projections to the upper limb are likely to exhibit a stronger relation between right-left FA asymmetry and right-left asymmetry in dexterous hand use. Tractography could help to distinguish corticospinal projections originating from the motor hand area from other descending projections and clarify whether the link between structural asymmetry and manual proficiency is specifically expressed in the corticospinal fibers conveying the descending motor commands to the hand.

Our study had a cross-sectional design covering an age range from 11 to 16 years. We observed a general improvement in the temporal regularity of circle drawing for both, left and right hand. Accordingly, the relation between both hands in terms of motor performance remained unchanged and no significant age effect on rightleft asymmetry was seen. With respect to DTI-based microstructural measures, we found a relative decrease in MD and increase in FA with age, which fits well with previous reports (Eluvathingal et al. 2007; Lebel et al. 2008; Madsen et al. 2011). With respect to right-left asymmetry, the relation between MD values in left and right CST was comparable across the age range, suggesting a symmetrical maturation. In contrast, FA values exhibited an age effect only in the left CST but not in the right CST. This divergence is in agreement with a previous study showing that age-dependent increases in FA-values may diverge for homologous tracts in the right and left hemisphere (Eluvathingal et al. 2007). However, a prospective longitudinal study is needed to properly clarify this issue. Here it would be interesting to start at pre-school age given the relatively protracted maturation of the CST during childhood and adolescence (Westlye et al. 2010) in order to obtain better insight into the developmental trajectories of manual proficiency.

Acknowledgments The HUBU project was supported by the Danish Council for Independent Research I Medical Sciences [Grant number 09-060166] and the Lundbeck Foundation [Grant number R32-A3161]. S.A. received financial support by the Faculty of Health Sciences, Copenhagen University. H.R.S received financial support from the Lundbeck Foundation [Grant of Excellence "Mapping, Modulation and Modeling the Control of Actions"; Grant number R59-A5399] and the Novo Nordisk Foundation Interdisciplinary Synergy Program 2014 ["Biophysically adjusted state-informed cortex stimulation (BASICS)"; Grant number NNF14OC0011413]. K.S.M received financial support by the Danish Medical Research Council [Grant Number 0602-02099B].

\section{Compliance with ethical standards}

Conflict of interest H.R.S. has served on a scientific advisory board for Lundbeck A/S, Valby Denmark, and has received honoraria as speaker from Biogen Idec, Denmark A/S, Genzyme, Denmark and MerckSerono, Denmark, has received honoraria as editor from Elsevier Publishers, Amsterdam, The Netherlands and Springer Publishing, Stuttgart, Germany, has received travel support from MagVenture, Denmark, and has received a research fund from Biogen-idec. All other authors declare that they have no conflict of interest.

Open Access This article is distributed under the terms of the Creative Commons Attribution 4.0 International License (http://crea tivecommons.org/licenses/by/4.0/), which permits unrestricted use, distribution, and reproduction in any medium, provided you give appropriate credit to the original author(s) and the source, provide a link to the Creative Commons license, and indicate if changes were made.

\section{References}

Amunts K, Jancke L, Mohlberg H, Steinmetz H, Zilles K (2000) Interhemispheric asymmetry of the human motor cortex related to handedness and gender. Neuropsychologia 38(3):304-312

Andersson JLR, Hutton C, Ashburner J, Turner R, Friston K (2001) Modeling geometric deformations in EPI time series. Neuroimage 13(5):903-919

Andersson JL, Jenkinson M, Smith S, Andersson J (2007). Non-linear registration, aka spatial normalization: FMRIB technical report TR07JA2. FMRIB Analysis Group of the University of Oxford

Barkovich AJ (2000) Concepts of myelin and myelination in neuroradiology. Am J Neuroradiol 21(6):1099-1109

Basser P, Mattiello J, LeBihan D (1994) MR diffusion tensor spectroscopy and imaging. Biophys J 66(1):259-267 
Beaulieu C (2002) The basis of anisotropic water diffusion in the nervous system - a technical review. NMR Biomed $15(7-8): 435-455$

Beaulieu C (2009) The biological basis of diffusion anisotropy. In: Behrens TEJ, Johansen-Berg $\mathrm{H}$ (eds) Diffusion MRI: from quantitative measurement to in vivo neuroanatomy. Elsevier, London, pp 105-126

Blair RC, Karniski W (1993) An alternative method for significance testing of waveform difference potentials. Psychophysiology 30(5):518-524

Buchel C, Raedler T, Sommer M, Sach M, Weiller C, Koch MA (2004) White matter asymmetry in the human brain: a diffusion tensor MRI study. Cereb Cortex 14(9):945-951

Damoiseaux JS, Greicius MD (2009) Greater than the sum of its parts: a review of studies combining structural connectivity and resting-state functional connectivity. Brain Struct Funct 213(6):525-533

Dassonville P, Zhu XH, Ugurbil K, Kim SG, Ashe J (1997) Functional activation in motor cortex reflects the direction and the degree of handedness. Proc Natl Acad Sci USA 94(25): 14015-14018

Dubois J, Dehaene-Lambertz G, Soarès C, Cointepas Y, Le Bihan D, Hertz-Pannier L (2008) Microstructural correlates of infant functional development: example of the visual pathways. J Neurosci 28(8):1943-1948

Eluvathingal TJ, Hasan KM, Kramer L, Fletcher JM, Ewing-Cobbs L (2007) Quantitative diffusion tensor tractography of association and projection fibers in normally developing children and adolescents. Cereb Cortex 17(12):2760-2768

Friederici AD (2009) Pathways to language: fiber tracts in the human brain. Trends Cogn Sci 13(4):175-181

Gong G, Jiang T, Zhu C, Zang Y, He Y, Xie S, Xiao J (2005) Side and handedness effects on the cingulum from diffusion tensor imaging. Neuroreport 16(15):1701-1705

Good CD, Johnsrude I, Ashburner J, Henson RNA, Friston KJ, Frackowiak RSJ (2001) Cerebral asymmetry and the effects of sex and handedness on brain structure: a voxel-based morphometric analysis of 465 normal adult human brains. Neuroimage 14(3):685-700

Groppa S, Werner-Petroll N, Münchau A, Deuschl G, Ruschworth MFS, Siebner HR (2012) A novel dual-site transcranial magnetic stimulation paradigm to probe fast facilitatory inputs from ipsilateral dorsal premotor cortex to primary motor cortex. Neuroimage 62(1):500-509

Groppe DM, Urbach TP, Kutas M (2011) Mass univariate analysis of event-related brain potentials/fields I: a critical tutorial review. Psychophysiology 48(12):1711-1725

Gulani V, Webb AG, Duncan ID, Lauterbur PC (2001) Apparent diffusion tensor measurements in myelin-deficient rat spinal cords. Magn Reson Med 45(2):191-195

Hartline DK, Colman DR (2007) Rapid conduction and the evolution of giant axons and myelinated fibers. Curr Biol 17(1):R25-R39

Hasan KM, Narayana PA (2006) Retrospective measurement of the diffusion tensor eigenvalues from diffusion anisotropy and mean diffusivity in DTI. Magn Reson Med 56(1):130-137

Healey JM, Liederman J, Geschwind N (1986) Handedness is not a unidimensional trait. Cortex 22(1):33-53

Henkel V, Mergl R, Juckel G, Rujescu D, Mavrogiorgou P, Giegling I, Moller $\mathrm{H}$ et al (2001) Assessment of handedness using a digitizing tablet: a new method. Neuropsychologia 39(11):1158-1166

Herve PY, Crivello F, Perchey G, Mazoyer B, Tzourio-Mazoyer N (2006) Handedness and cerebral anatomical asymmetries in young adult males. Neuroimage 29(4):1066-1079

Herve PY, Leonard G, Perron M, Pike B, Pitiot A, Richer L, Veillette $S$ et al (2009) Handedness, motor skills and maturation of the corticospinal tract in the adolescent brain. Hum Brain Mapp 30(10):3151-3162

Hua K, Zhang J, Wakana S, Jiang H, Li X, Reich DS, Calabresi PA et al (2008) Tract probability maps in stereotaxic spaces: analyses of white matter anatomy and tract-specific quantification. Neuroimage 39(1):336-347

Janssen JP (2004) Evaluation of empirical methods and methodological foundations of human left-handedness. Percept Motor Skill 98(2):487-506

Jito J, Nakasu S, Ito R, Fukami T, Morikawa S, Inubushi T (2008) Maturational changes in diffusion anisotropy in the rat corpus callosum: comparison with quantitative histological evaluation. JMRI-J Magn Reson Imaging 28(4):847-854

Jovicich J, Czanner S, Greve D, Haley E, van der Kouwe A, Gollub R, Kennedy D et al (2006) Reliability in multi-site structural MRI studies: effects of gradient non-linearity correction on phantom and human data. Neuroimage 30(2):436-443

Jung P, Klein JC, Wibral M, Hoechstetter K, Bliem B, Lu MK, Wahl $M$ et al (2012) Spatiotemporal dynamics of bimanual integration in human somatosensory cortex and their relevance to bimanual object manipulation. J Neurosci 32(16):5667-5677

Klarborg B, Skak Madsen K, Vestergaard M, Skimminge A, Jernigan TL, Baaré WFC (2013) Sustained attention is associated with right superior longitudinal fasciculus and superior parietal white matter microstructure in children. Hum Brain Mapp 34(12):3216-3232

Kloppel S, Vongerichten A, van Eimeren T, Frackowiak RS, Siebner HR (2007) Can left-handedness be switched? Insights from an early switch of handwriting. J Neurosci 27(29):7847-7853

Kumar A, Juhasz C, Asano E, Sundaram SK, Makki MI, Chugani DC, Chugani HT (2009) Diffusion tensor imaging study of the cortical origin and course of the corticospinal tract in healthy children. Am J Neuroradiol 30(10):1963-1970

Lebel C, Walker L, Leemans A, Phillips L, Beaulieu C (2008) Microstructural maturation of the human brain from childhood to adulthood. Neuroimage 40(3):1044-1055

Lemon RN (1999) Neural control of dexterity: what has been achieved? Exp Brain Res 128(1-2):6-12

Lemon RN (2008) Descending pathways in motor control. Annu Rev Neurosci 31:195-218

Li L, Preuss TM, Rilling JK, Hopkins WD, Glasser MF, Kumar B, Nana R et al (2010) Chimpanzee (Pan troglodytes) precentral corticospinal system asymmetry and handedness: a diffusion magnetic resonance imaging study. PLoS One 5(9):e12886

Madsen KS, Baare WFC, Vestergaard M, Skimminge A, Ejersbo LR, Ramsoy TZ, Gerlach C et al (2010) Response inhibition is associated with white matter microstructure in children. Neuropsychologia 48(4):854-862

Madsen KS, Baare WFC, Skimminge A, Vestergaard M, Siebner HR, Jernigan TL (2011) Brain microstructural correlates of visuospatial choice reaction time in children. Neuroimage 58(4):1090-1100

Marquardt C, Mai N (1994) A computational procedure for movement analysis in handwriting. J Neurosci Meth 52(1):39-45

Mori S, Wakana S, Nagae-Poetscher LM, Van Zijl PCM (2005) MRI atlas of human white matter. Elsevier, Amsterdam

Mukherjee P, Miller JH, Shimony JS, Philip JV, Nehra D, Snyder AZ, Conturo TE et al (2002) Diffusion-tensor MR imaging of gray and white matter development during normal human brain maturation. Am J Neuroradiol 23(9):1445-1456

Nichols TE, Holmes AP (2002) Nonparametric permutation tests for functional neuroimaging: a primer with examples. Hum Brain Mapp 15(1):1-25

Ocklenburg S, Güntürkün O (2012) Hemispheric asymmetries: the comparative view. Front Psychol 3:5

Oldfield RC (1971) Assessment and analysis of handednessEdinburgh inventory. Neuropsychologia 9(1):97-113 
Reese TG, Heid O, Weisskoff RM, Wedeen VJ (2003) Reduction of eddy-current-induced distortion in diffusion MRI using a twicerefocused spin echo. Magn Reson Med 49(1):177-182

Rose S, Rowland T, Pannek K, Baumann F, Coulthard A, Mccombe P, Henderson R (2012) Structural hemispheric asymmetries in the human precentral gyrus hand representation. Neuroscience 210:211-221

Seizeur R, Magro E, Prima S, Wiest-Daessle N, Maumet C, Morandi $\mathrm{X}$ (2013) Corticospinal tract asymmetry and handedness in rightand left-handers by diffusion tensor tractography. Surg Radiol Anat 36(2):111-124

Siebner HR, Limmer C, Peinemann A, Drzezga A, Bloem BR, Schwaiger M, Conrad B (2002) Long-term consequences of switching handedness: a positron emission tomography study on handwriting in "converted" left-handers. J Neurosci 22(7):2816-2825

Smith SM, Nichols TE (2009) Threshold-free cluster enhancement: addressing problems of smoothing, threshold dependence and localisation in cluster inference. Neuroimage 44(1):83-98

Smith SM, Jenkinson M, Johansen-Berg H, Rueckert D, Nichols TE, Mackay CE, Watkins KE et al (2006) Tract-based spatial statistics: voxelwise analysis of multi-subject diffusion data. Neuroimage 31(4):1487-1505

Song SK, Yoshino J, Le TQ, Lin SJ, Sun SW, Cross AH, Armstrong RC (2005) Demyelination increases radial diffusivity in corpus callosum of mouse brain. Neuroimage 26(1):132-140

Sun ZY, Kloppel S, Riviere D, Perrot M, Frackowiak R, Siebner HR, Mangin JF (2012) The effect of handedness on the shape of the central sulcus. Neuroimage 60(1):332-339

Vernooij MW, Smits M, Wielopolski PA, Houston GC, Krestin GP, van der Lugt A (2007) Fiber density asymmetry of the arcuate fasciculus in relation to functional hemispheric language lateralization in both right- and left-handed healthy subjects: a combined fMRI and DTI study. Neuroimage 35(3):1064-1076

Vestergaard M, Madsen KS, Baare WFC, Skimminge A, Ejersbo LR, Ramsøy TZ, Gerlach C, Åkeson P, Paulson OB, Jernigan TL (2011) White matter microstructure in superior longitudinal fasciculus associated with spatial working memory performance in children. J Cogn Neurosci 23(9):2135-2146

Volkmann J, Schnitzler A, Witte OW, Freund HJ (1998) Handedness and asymmetry of hand representation in human motor cortex. J Neurophysiol 79(4):2149

Watkins KE, Paus T, Lerch JP, Zijdenbos A, Collins DL, Neelin P, Taylor J et al (2001) Structural asymmetries in the human brain: a voxel-based statistical analysis of 142 MRI scans. Cereb Cortex 11(9):868

Westerhausen R, Huster RJ, Kreuder F, Wittling W, Schweiger E (2007) Corticospinal tract asymmetries at the level of the internal capsule: is there an association with handedness? Neuroimage 37(2):379-386

Westlye LT, Walhovd KB, Dale AM, Bjornerud A, Due-Tonnessen P, Engvig A, Grydeland $\mathrm{H}$ et al (2010) Life-span changes of the human brain white matter: diffusion tensor imaging (DTI) and volumetry. Cereb Cortex 20(9):2055-2068

Wolbers T, Schoell ED, Buchel C (2006) The predictive value of white matter organization in posterior parietal cortex for spatial visualization ability. Neuroimage 32(3):1450-1455

Yagishita A, Nakano I, Oda M, Hirano A (1994) Location of the corticospinal tract in the internal capsule at MR imaging. Radiology 191:455-460

Yousry TA, Schmid UD, Alkadhi H, Schmidt D, Peraud A, Buettner A, Winkler P (1997) Localization of the motor hand area to a knob on the precentral gyrus. A new landmark. Brain 120(1):141-157 